WORLD VIEW

\title{
Outcomes of high volume cataract surgeries in a developing country
}

\author{
R Venkatesh, R Muralikrishnan, Linda Civerchia Balent, S Karthik Prakash, N Venkatesh Prajna
}

Br J Ophthalmol 2005;89:1079-1083. doi: 10.1136/bjo.2004.063479

See end of article for authors' affiliations ....................

Correspondence to: Linda Civerchia Balent, $M D$, Eye Care and Surgery Center of Fort Lauderdale, 1110 SE 4 th Street, Ft Lauderdale, FL 33301, USA; dralvan@msn.com

Accepted for publication 6 February 2005 lume cataract surgery in a developing country, community based, Aim: To analyse the outco
high volume eye hospital.

Methods: In a non-comparative interventional case series, the authors reviewed the surgical outcomes of 593 patients with cataract operated upon by three high volume surgeons on six randomly selected days. There were 318 female (54\%) and 275 male (46\%) patients. Their mean age was 59.57 (SD 10.13) years. The majority of the patients underwent manual small incision cataract surgery (manual SICS). Extracapsular cataract extraction with posterior chamber intraocular lens (ECCE-PCIOL) and intracapsular cataract extraction (ICCE) were also done on a few patients as clinically indicated.

Results: Best corrected visual acuity of $\geqslant 6 / 18$ was achieved in $94 \%$ of the 520 patients who could be followed up on the 40th postoperative day ( $88 \%$ follow up rate). Intraoperative and immediate postoperative complications as defined by OCTET occurred in $11(1.9 \%)$ and $75(12.6 \%)$ patients, respectively. Average surgical time of 3.75 minutes per case (16-18 cases per hour) was achieved. Statistically significant risk factors for outcomes were found to be age $>60$, sex, and surgeon.

Conclusion: High volume surgery using appropriate techniques and standardised protocols does not compromise quality of outcomes.
O $\mathrm{f}$ the total estimated 38 million blind people in the world, 9-12 million are in India. ${ }^{12}$ Estimates report that $50 \%-80 \%$ of these people are blind because of cataract. $^{12}$ In addition to the backlog, an additional 3.8 million become blind each year because of cataract. ${ }^{3}$ In 2000 , 3.5 million cataract operations were performed, ${ }^{4}$ but this remains insufficient to treat the backlog and the newly blind. In 1994, Natchiar et al suggested that productivity per individual surgeon/unit should be increased through a high volume, high quality cataract surgery approach to solve the problem of India's curable blind. ${ }^{5}$ More recently, the World Health Organization global initiatives have called for a dramatic increase in surgical volumes worldwide. ${ }^{6}$ However, it is becoming more evident that outcomes of cataract surgery are not always good and this aspect of surgical services must be further examined. ${ }^{7}$ Some recent studies of high volume cataract surgery in India and Nepal report good results. ${ }^{8-14}$ In order to investigate whether high volume surgeries can be performed routinely, without compromising quality, we retrospectively reviewed the results of 593 surgeries performed by three experienced surgeons, achieving surgical times of 3.75 minutes/case (16-18 cases/hour), utilising the technique of manual small incision cataract surgery (manual SICS).

\section{PATIENTS AND METHODS}

Surgeons consistently performing more than 80 surgeries per day in six operating hours at Aravind were defined as "high volume surgeons." There were six such high volume surgeons during the period of analysis from April 2002-March 2003. From these six surgeons, three were randomly chosen using a lot method. There were 28 days (clusters) during the year in which these surgeons had done more than 80 surgeries/day. A total of 2795 cataract surgeries were performed on these 28 high volume days. A sample size requirement of 550 cataract surgeries was based on estimating with $95 \%$ confidence, a cumulative complications (trivial, intermediate, and serious) rate and poor visual outcomes within the 40th day postoperative examination of $10 \%$ (plus or minus $3.0 \%$ ), including an anticipated 15\% loss to follow up and cluster sampling design effect of 1.5. The cumulative prevalence of complications and poor outcomes was based on previous literature. ${ }^{9}$

In order to achieve the required sample size, 2 days (clusters) were picked randomly from each study surgeon's high volume surgery days using lots. A total of 593 surgeries were done on these six randomly selected days (clusters), which was more than the estimated sample required. Possible risk factors that might affect outcomes such as age, sex, eye, preoperative visual acuity, and surgeon were recorded on a separate proforma. Further stratification of surgeries on each day as chronological quarters was used as a proxy measure to denote time of surgery.

All patients who underwent surgery were screened at various community based screening camps and transported to the base hospital according the system described by Natchair et al. ${ }^{5}$ Patient flow from screening to the 40th postoperative day is described in figure 1. In all, 582 patients (98\%) underwent manual SICS. Extracapsular cataract extraction with posterior chamber intraocular lens (ECCEPCIOL) was performed on nine patients $(1.5 \%)$ and two patients $(0.5 \%)$ underwent intracapsular cataract extraction (ICCE) as clinically indicated.

Manual SICS was performed with a $6-6.5 \mathrm{~mm}$ scleral incision made $2.5 \mathrm{~mm}$ behind the anterior limbus. A partial thickness scleral tunnel was dissected into the cornea with a crescent knife. A side port paracentesis was made to facilitate intraocular manipulation. The anterior chamber was entered with a $3.2 \mathrm{~mm}$ keratome and the internal lip of the incision was further extended. The anterior chamber was filled with

Abbreviations: BCVA, best corrected visual acuity; deff, design effects; ECCE, extracapsular cataract extraction; ICCE, intracapsular cataract extraction; OCTET, Oxford Cataract Treatment Evaluation Team; PCIOL, posterior chamber intraocular lens; PL, perception of light; SICS, small incision cataract surgery; UCVA, uncorrected visual acuity 


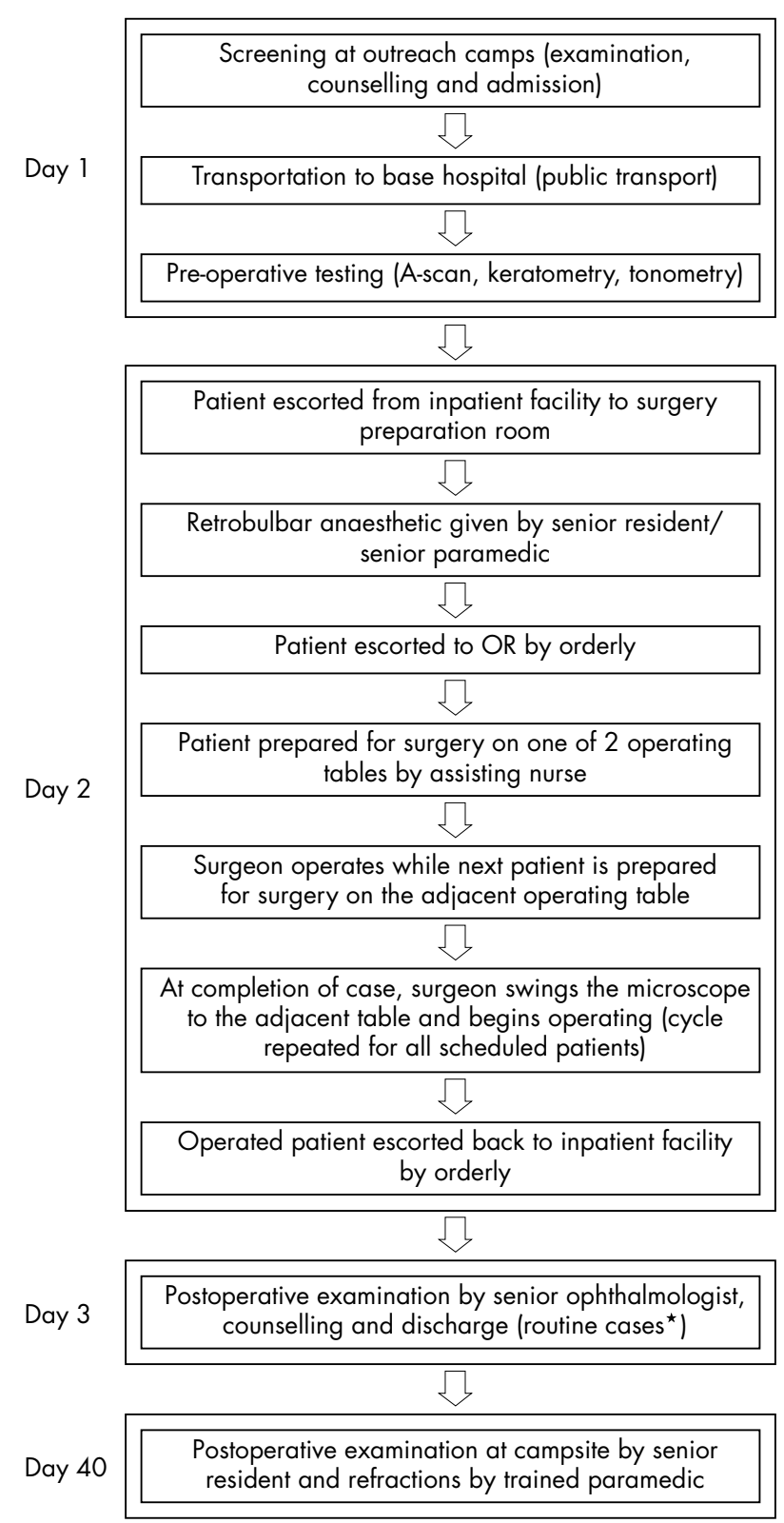

*Patients with complications are discharged when clinically indicated

Figure 1 Flow of patients for high volume surgery.

viscoelastic before making a $6-6.5 \mathrm{~mm}$ capsulorhexis. Canopener capsulotomy was performed in cases where the capsulorhexis extended peripherally. Hydrodissection and delineation of nucleus was performed to hydroprolapse the endonucleus into the anterior chamber. In cases of canopener capsulotomy, or where hydroprolapse is incomplete, a Sinsky hook was used to spin the nucleus into the anterior chamber. The nucleus was hydro-extracted with an irrigating vectis. The epinucleus and residual cortex were aspirated with a Simcoe canula and a $6 \mathrm{~mm}$ optic PMMA IOL (Aurolab, Madurai, India) was implanted. Contraindications to manual SICS include doubtful zonular integrity as a result of pseudoexfoliation, decompensated cornea, and hard nuclear cataract (>grade IV). Surgical protocols, well reported in the literature for ECCE-PCIOL and ICCE ${ }^{9}$ were followed. Protocols for the management of intraoperative complications were as follows. Patients with posterior capsule rupture or zonular dialysis without vitreous loss underwent IOL implantation in the ciliary sulcus. In patients with vitreous loss, anterior vitrectomy was done manually then the IOL was sulcus fixed. If stable IOL fixation could not be obtained, the patient was left aphakic.

Patient flow in the operating room followed a modification of the system reported by Natchiar et al. ${ }^{5}$ This system, originally designed for ICCE and ECCE-IOL surgery, had to be modified to accommodate the speed of manual SICS (fig l). Operating room personnel includes the surgeon, three assisting nurses, one orderly, one circulating nurse, and one nurse to sterilise the instruments. The surgeon operates with one microscope set to swing between two tables. The assisting nurses prepare the patient for surgery by draping and placing the superior rectus bridle suture. The third assisting nurse helps the others to arrange sterilised instruments and pack unsterile instruments for autoclaving. The circulating nurse supplies consumables to the assisting nurses, completes the case record, and bandages the operated eye. At the completion of surgery, the surgeon injects subconjunctival antibiotic and steroid. Intraocular fluids are not changed between cases and only the Simcoe cannula is changed for each surgery. In between the surgeries, the surgeon rinses his gloved hands with chlorhexidine antiseptic solution. Five sets of surgical instruments (each consisting of 15 instruments) are assigned to each table (total 10 sets). All instruments are sterilised in a Flash autoclave in which 4-5 sets were run for 15 minutes. In this high volume system, normal cases are scheduled first. Complicated cases are scheduled later in the day (the fourth quarter), as they often do not proceed routinely.

Intraoperative and immediate postoperative complications were categorised using Oxford Cataract Treatment Evaluation Team (OCTET) ${ }^{15}$ definitions. The preoperative, intraoperative, and postoperative findings and complications were recorded on a programmed case sheet. Levels of visual acuity after cataract surgery were categorised using the WHO guidelines-that is, good outcome was defined as 6/6-6/18, borderline outcome as 6/24-6/60, and poor outcome as $<6 / 60$.

Data were analysed using Stata 7.0 version. Association of risk factors with outcomes was assessed by multiple logistic regression analysis. Confidence intervals (CI) for prevalence estimates and for odds ratio from the regression analysis was calculated taking design effects (deff) associated with cluster sampling design into account. institutional review board/

Table 1 Distribution of surgeries according to surgeons and random days

\begin{tabular}{llll}
\hline \multirow{2}{*}{ Surgeon } & \multicolumn{2}{l}{ Random day } & \\
\cline { 2 - 3 } & Day 1 & Day 2 & Total \\
\hline 1 & $97(33 \%)$ & $96(32.5 \%)$ & $193(32 \%)$ \\
2 & $95(32 \%)$ & $96(32.5 \%)$ & $191(33 \%)$ \\
3 & $105(35 \%)$ & $104(35 \%)$ & $209(35 \%)$ \\
Total & $297(100 \%)$ & $296(100 \%)$ & $593(100 \%)$ \\
\hline
\end{tabular}


Table 2 Distribution of intraoperative complications

\begin{tabular}{llc}
\hline Complication & $\begin{array}{l}\text { OCTET } \\
\text { grading* }\end{array}$ & No (\%) \\
\hline $\begin{array}{l}\text { Posterior capsule rupture (with no vitreous loss) } \\
\text { Posterior capsule rupture (with vitreous loss) }\end{array}$ & 4 & $4(0.7)$ \\
Zonular dialysis (without vitreous loss) & III & $11(1.9)$ \\
Total & & $(95 \% \mathrm{Cl}:-0.11 \%$ to \\
& $3.82 \%$, deff 1.910$)$ \\
Total surgeries & $593(100)$ \\
\hline *Grade I, trivial complications that may have needed medical therapy, but were not likely to result in a marked \\
drop in visual acuity; grade II, intermediate complications that needed medical therapy and would have resulted in \\
a marked drop in visual acuity if left untreated; grade III, serious complications that would have needed immediate \\
medical or surgical intervention to prevent gross visual loss.
\end{tabular}

ethics committee approval was not required as the study was reporting what happens on a routine day.

\section{RESULTS}

Within the six randomly selected days (six operating hours per day), three surgeons performed a total of 593 cataract surgeries. Table 1 presents the number of surgeries done by the three surgeons on each randomly selected day with no statistically significant difference $(p=0.993)$.

The average time per surgery was around 3.75 minutes (16-18 cases/hour). In all, 526 patients $(88.5 \%)$ had a preoperative uncorrected visual acuity between perception of light (PL) and 5/60, 65 patients (11\%) between 6/60 and $6 / 24$, and two patients $(0.5 \%)$ had preoperative visual acuity of $6 / 18$ (fig 2 ).

\section{Intraoperative complications}

Of the 593 patients, 11 (1.9\%) had intraoperative complications (table 2). Further on multiple logistic regressions, none of the risk factors had associations with intraoperative complication except surgeon (table 3). Two of the three surgeons had intraoperative complications. The odds ratio for surgeon 2 compared to surgeon 3 was 2.83 (deff 0.167).

\section{Immediate postoperative complications}

Of the 593 patients, $526(89 \%)$ patients did not have any immediate postoperative complications on first day follow up examination. Sixty seven eyes had 75 complications (12.6\%).
According to OCTET grading, 55 patients $(9.2 \%)$ had grade I complications, 19 patients (3.2\%) had grade II complications, and one patient $(0.2 \%)$ had a grade III complication (table 4$)$.

Postoperative complications were managed as follows. Severe iritis was treated with frequent use of topical steroids. One patient with hypopyon received subconjunctival garamycin and dexamethsone along with frequent topical steroids. In patients with haptic malposition, the intraocular lens was redialed on the first postoperative day. Other complications resolved with routine postoperative medical therapy.

On multiple logistic regressions, significant risk associations were found with age and surgeons (table 3 ). Patients above 60 years were 1.67 times (deff 0.343 ) more likely to have postoperative complications. Surgeon 2 and 3 were 4.99 (deff 0.086) and 2.10 (deff 0.197) times more likely to have postoperative complications respectively, than surgeon I (table 3). Interaction between age and surgeons were also further investigated and the variables were found to be valid.

\section{Postoperative visual acuity}

Seventy three patients (12\%) were lost to follow up on the 40th postoperative day. Of the 520 patients followed up, UCVA was $>6 / 18$ for 228 (43.9\%) patients, $6 / 24$ to $6 / 60$ for $265(51 \%)$ patients and lesser than $6 / 60$ for $27(5.3 \%)$ patients (table 4). A total of 491 patients $(94.4 \%)$ achieved BCVA of $>6 / 18,21$ patients $(4 \%)$ achieved $6 / 24$ to $6 / 60$ and eight patients $(1.6 \%$ ) had $<6 / 60$ (table 5 ). In patients with poor

Table 3 Odds ratio $(95 \% \mathrm{Cl}$, p value) on multiple logistic regression for outcomes

\begin{tabular}{|c|c|c|c|c|}
\hline & \multicolumn{2}{|l|}{ Operative complications } & \multicolumn{2}{|c|}{ Postoperative visual acuity $(<6 / 18)$} \\
\hline & Intraoperative $(n=11 / 593)$ & Postoperative ( $n=75 / 593$ ) & UCVA $(n=292 / 520)$ & BCVA $(n=29 / 520)$ \\
\hline \multicolumn{5}{|l|}{ Age } \\
\hline$\leqslant 60$ & 1.0 & 1.0 & 1.0 & 1.0 \\
\hline$>60$ & $2.29(0.85$ to $61.72,0.483)$ & $1.67(1.13$ to $2.47,0.020)$ & $1.65(0.98$ to $2.77,0.056)$ & $1.50(0.58$ to $3.91,0.324)$ \\
\hline \multicolumn{5}{|c|}{ 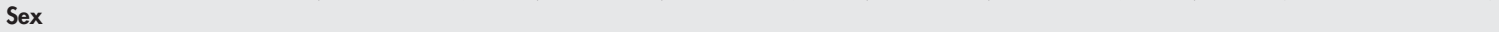 } \\
\hline Female & 1.0 & 1.0 & 1.0 & 1.0 \\
\hline Male & $0.95(0.20$ to $4.57,0.917)$ & $0.97(0.55$ to $1.70,0.882)$ & $0.92(0.43$ to $1.98,0.810)$ & $2.38(1.39$ to $4.05,0.009)$ \\
\hline \multicolumn{5}{|c|}{0.72 p.40 } \\
\hline Right & 1.0 & 1.0 & 1.0 & 1.0 \\
\hline Left & $3.33(0.59$ to $18.68,0.113)$ & 1.05 (0.34 to $3.24,0.924)$ & $1.22(0.72$ to $2.04,0.369)$ & $1.14(0.32$ to $4.09,0.805)$ \\
\hline \multicolumn{5}{|l|}{ Preop VA } \\
\hline$<6 / 60$ & None & $1.51(0.37$ to $6.13,0.482)$ & $1.02(0.64$ to $1.63,0.913)$ & $2.17(0.25$ to $19.18,0.401)$ \\
\hline \multicolumn{5}{|c|}{ 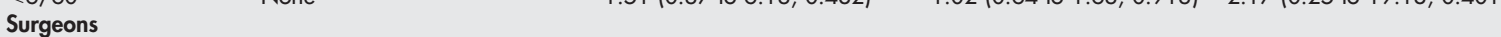 } \\
\hline 1 & None & 1.0 & 1.0 & 1.0 \\
\hline 2 & $2.83(1.15$ to $6.91,0.034)$ & $4.99(3.81$ to $6.53,<0.001)$ & 1.14 (0.74 to $1.74,0.454)$ & $1.34(0.71$ to $2.53,0.287)$ \\
\hline 3 & 1.0 & $2.10(1.36$ to $3.24,0.007)$ & $0.54(0.24$ to $1.25,0.121)$ & $0.84(0.45$ to $1.54,0.495)$ \\
\hline \multicolumn{5}{|c|}{ Time of surgery } \\
\hline 1st quarter & 1.0 & 1.0 & 1.0 & 1.0 \\
\hline 2nd quarter & $1.63(0.32$ to $8.22,0.403)$ & $1.82(0.62$ to $5.38,0.215)$ & $1.46(0.53$ to $4.07,0.385)$ & $0.76(0.55$ to $10.54,0.804)$ \\
\hline $3 r d$ quarter & $0.71(0.29$ to $17.60,0.763)$ & $1.78(0.63$ to $5.04,0.215)$ & $1.95(0.78$ to $4.90,0.120)$ & $1.31(0.12$ to $14.83,0.780)$ \\
\hline 4th quarter & $1.09(0.40$ to $30.21,0.937)$ & $1.72(0.52$ to $5.65,0.295)$ & $1.82(0.58$ to $5.68,0.233)$ & $1.63(0.13$ to $20.60,0.640)$ \\
\hline
\end{tabular}


Table 4 Distribution of postoperative complications

\begin{tabular}{|c|c|c|}
\hline Complication & $\begin{array}{l}\text { OCTET } \\
\text { grading* }\end{array}$ & No (\%) \\
\hline Hypopyon & III & $1(0.2)$ \\
\hline Severe iritis (>50 cells in $2 \times 1 \mathrm{~mm}$ slit beam) & $\|$ & $19(3.2)$ \\
\hline Mild iritis ( $<50$ cells in $2 \times 1 \mathrm{~mm}$ slit beam) & I & $16(2.7)$ \\
\hline Transient corneal oedema & I & $9(1.5)$ \\
\hline Transient corneal oedema, Descement's membrane folds $<10$ & i & $19(3.2)$ \\
\hline Transient corneal oedema, Descement's membrane folds $>10$ & I & $5(0.8)$ \\
\hline Hyphaema $<3 \mathrm{~mm}$ blood in anterior chamber & 1 & $1(0.2)$ \\
\hline Residual cortex & I & $1(0.2)$ \\
\hline Vitreous in anterior chamber not touching cornea & I & $1(0.2)$ \\
\hline Malposition of haptic & I & $3(0.5)$ \\
\hline Total & & 75 (12.6) \\
\hline & & $\begin{array}{l}\text { ( } 95 \% \text { Cl: } 4.85 \% \text { to } 20.45 \% \text {, deff } \\
4.934 \text { ) }\end{array}$ \\
\hline Total surgeries & & $593(100)$ \\
\hline
\end{tabular}

visual acuity, the most common reason was pre-existing ocular pathology.

On a multiple logistic regression analysis, there was no statistically significant association between risk factors and postoperative UCVA (table.3). On examination of risk factors and BCVA, there was a statistically significant association with the sex of the patient (table 3). Male patients were likely to have a risk (odds ratio $2.38,95 \%$ CI, 1.39 to 4.05 , deff 0.229 ) of achieving BCVA $<6 / 18$.

\section{DISCUSSION}

Our UCVA results (43.9\%), compare favourably with a recent randomised trial comparing ECCE and manual SICS in India. ${ }^{16}$ The difference between UCVA and BCVA brings out the fact that residual refractive error is a major deterrent to successful outcomes. Measurements of visual acuity and refractions at large campsites are difficult because of suboptimal facilities in relation to chart illumination and refraction area, patient volume, lack of patient comprehension, and difficulties in data recovery. Our study focus was to determine operative and postoperative complication rates and surgical risk factors in high volume surgery rather than to study refractive outcomes. Refractive data from the campsite were often inadquate, but it appeared that surgically induced

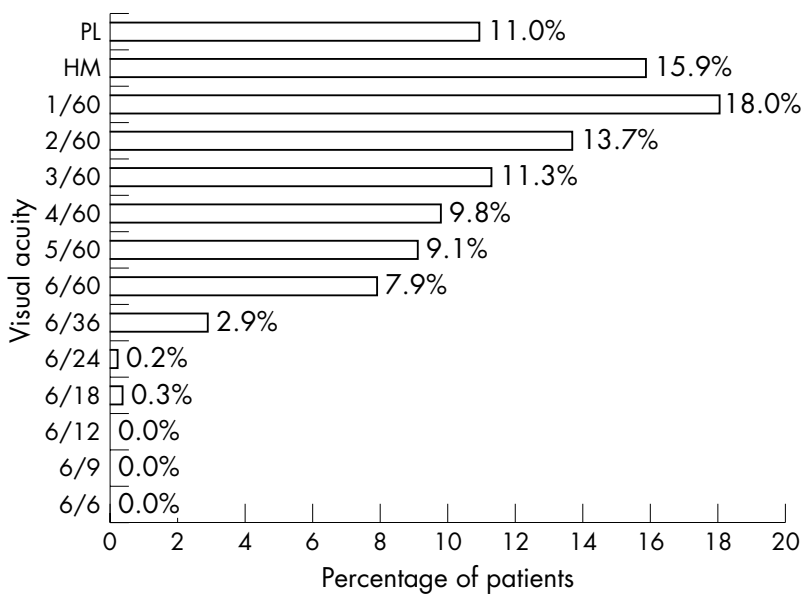

Figure 2 Distribution of patients and preoperative uncorrected visual acuity $(n=593)$. against the rule astigmatism was the main reason for decreased UCVA compared to BCVA. However, considering the patient's preoperative acuity status $(88.5 \%$ of patients between perception of light and 5/60), this visual outcome is a marked improvement. Recently, Hennig et al reported higher rates of UCVA $>6 / 18^{14}$ with manual SICS. Although this study in Nepal excluded cases with pre-existing ocular pathology, which we did not, this might warrant some modification in our own technique, which has an advantage of increased surgical throughput (16-18 cases per hour) and significantly less hyphaema. Combining these findings is of critical importance in the global effort to eliminate cataract blindness.

The BCVA results in our study compare favourably with results reported from other studies on manual SICS, ${ }^{8} 91416$ standard extracapsular surgery, ${ }^{11-13}{ }^{16}$ and instrumental phacoemulsification. ${ }^{17}{ }^{18}$ The BCVA results also compare favourably with the meta-analysis results of 90 studies on cataract surgeries done in the United States and reported by Powe et al. ${ }^{19}$

Intraoperative complications were present in $1.9 \%$ of the study patients, which is similar to other manual SICS studies. $^{8}{ }^{916}$ The postoperative complication of manual SICS in our study compares favourably with the results reported in other manual SICS, ${ }^{8}{ }^{16}$ ECCE/PC-IOL, ${ }^{11}{ }^{12} 16$ and instrumental phacoemulsification ${ }^{17} 18$ studies.

Our results report a statistically significant difference between surgeons with intraoperative and postoperative complications. Though the complication rate in our study was low, this variability among surgeons has been reported elsewhere and is probably not avoidable. ${ }^{10}{ }^{20}$ However, we cannot exclude the possibility that the surgeons with higher complication rates operated on more of the complicated cases.

Comparing this study with the previous randomised trial at our hospital-MIOL, ${ }^{10}$ outcomes like UCVA and BCVA are not nearly so favourable in the present study as in the MIOL trial. This is mainly because the MIOL trial had strict exclusion criteria $^{21}$ and the present study did not exclude any patients with ocular co-morbidity. Our design was adopted to demonstrate what happens on a routine basis rather than to present the efficacy of procedures. However, intraoperative and postoperative complication data in our study compare favourably with data from the MIOL extracapsular group. The overall infection rate at our hospital is one in 1000 cases (with no significant difference between high or low volume days) and is comparable with international standards. 
Table 5 Distribution of patients with postoperative uncorrected and best corrected visual acuity

\begin{tabular}{|c|c|c|}
\hline \multirow[b]{2}{*}{ VA } & \multicolumn{2}{|l|}{ No of patients (\%) } \\
\hline & UCVA & BCVA \\
\hline$\geqslant 6 / 18$ (good) & $\begin{array}{l}228 \quad(43.9) \\
(95 \% \text { Cl: } 32.30 \% \text { to } 55.40 \% \text {, deff } 4.255)\end{array}$ & $\begin{array}{l}491 \text { (94.4) } \\
(95 \% \text { Cl: } 92.47 \% \text { to } 96.37 \% \text { deff } 0.568)\end{array}$ \\
\hline 6/24-6/60 (borderline) & $\begin{array}{l}265 \text { (51) } \\
(95 \% \text { Cl: } 40.02 \% \text { to } 61.90 \% \text {, deff } 3.764)\end{array}$ & $\begin{array}{l}21 \quad(4.0) \\
(95 \% \mathrm{Cl}: 1.80 \% \text { to } 6.28 \% \text {, deff } 1.018)\end{array}$ \\
\hline$<6 / 60$ (poor) & $\begin{array}{l}27 \quad 15.3) \\
(95 \% \mathrm{Cl}: 1.17 \% \text { to } 9.21 \% \text {, deff } 2.577)\end{array}$ & $\begin{array}{l}8 \\
(95 \% \mathrm{Cl}: 0.07 \% \text { to } 3.00 \% \text { deff } 1.110)\end{array}$ \\
\hline Total & $520 \quad(100.0)$ & $520 \quad(100.0)$ \\
\hline
\end{tabular}

The average time per surgery was around 3.75 minutes (16-18 cases per hour). Other IOL studies have also reported surgical time of $12-16$ cases per hour. ${ }^{10}$ One of the limitations of our retrospective study is that we could not record the exact time for each surgery. This leaves a chance of bias because of the association of complications with increased length of the surgery. Other limitations of our study include the lack of complete preoperative and postoperative refractive data as they was not documented in many patients at the campsite. A prospective study with hospital based postoperative examination and refraction is recommended.

The study results shows that high quality cataract surgery (98\% without intraoperative complications, 94\% BCVA 6/18 or better) can be attained in a high volume setting. This is dependent on the choice of surgical technique (manual SICS), standardised protocols, standardised training of surgeons and paramedical personnel, and an overall organisational structure that supports high volume patient flow. This has significant implications to developing countries because the principal solution to the backlog of cataract blind is performing cataract operations on a large scale. Our study highlights the need to establish systems to monitor throughputs and quality of cataract surgery. These results are of significant relevance for the WHO's "Vision 2020" programme. $^{5}$

\section{Authors' affiliations}

R Venkatesh, N V Prajna, Aravind Eye Hospital, 1 Anna Nagar, Madurai-625020 Tamil Nadu, India

R Muralikrishnan, S K Prakash, Lions Aravind Institute of Community Ophthalmology, 72, Kuruvikaran Salai, Gandhi Nagar, Madurai-625 020 Tamil Nadu, India

L C Balent, Eye Care and Surgery Center of Fort Lauderdale, 1110 SE 4th Street, Ft Lauderdale, FL 33301, USA

\section{REFERENCES}

1 Jose R. National programme for control of blindness. Indian J Commun Health 1997;3:5-9.

2 Dandona L, Dandona R, Naduvilath T, et al. Is the current eye-care policy focus almost exclusively on cataract adequate to deal with blindness in India? Lancet 1998;351:1312-16.
3 Minassian D, Mehra V. 3. 8 Million blinded by cataract each year: projections from the first epidemiological study of incidence of cataract blindness in India, Br J Ophthalmol 1990;74:341-3.

4 Dandona L, Dandona R, Anand R, et al. Outcome and number of cataract surgeries in India: policy issues for blindness control. Clin Experiment Ophthalmol 2003:31:23-31.

5 Natchiar G, Robin AL, Ravilla D, et al. Attacking the backlog of India's curable blind. Arch Ophthalmol 1994;1 12:987-93.

6 Thylefors B. A global initiative for the elimination of avoidable blindness [editorial]. Am J Ophthalmol 1998;125:90-3.

7 Foster A. Cataract-a global perspective: output, outcome and outlay. Eye 1999;13:449-53

8 Natchiar G, DabralKar T. Manual small incision suture less cataract surgeryan alternative technique to instrumental phacoemulsification. Operative Techniques Cataract Refract Surg 2000;3:161-70.

9 Balent LC, Narendran K, Patel S, et al. High volume sutureless intraocular lens surgery in a rural eye camp in India. Ophthalmic Surg Lasers 2001;32:446-55

10 Prajna NV, Chandrakanth Ks, Kim R, et al. The Madurai intraocular lens study II: Clinical outcomes. Am J Ophthalmol 1998;125:14-25.

11 Civerchia L, Apoorvananda SW, Natchiar G, et al. Intraocular lens implantation in rural India. Ophthalmic Surg Lasers 1993;24:648-53.

12 Civerchia L, Ravindran RD, Apoorvananda SW, et al. High volume intraocular lens surgery in a rural eye camp in India. Ophthalmic Surg Lasers 1996;27:200-8.

13 Ruit S, Tabin GC, Nissman SA, et al. Low cost high volume extracapsular cataract extraction with posterior chamber intraocular lens implantation in Nepal. Ophthalmology 1999;106:1887-92.

14 Hennig A, Kumar J, Yorston D, et al. Sutureless cataract surgery with nucleus extraction: outcome of a prospective study in Nepal. Br J Ophthalmol 2003;87:266-70

15 Oxford Cataract Treatment and Evaluation Team. Use of grading system in evaluation of complications in a randomised controlled trial. Br J Ophthalmol 1986;70:411-14

16 Gogate PM, Deshpande M, Wormald RP, et al. Extracapsular cataract surgery compared with manual small incision cataract surgery in community eye care setting in western India: a randomised controlled trial. Br J Ophthalmol 2003;87:667-72.

17 David T, Rowe NA, Francis IC, et al. Intraoperative complications of 1000 phacoemulsification procedure: a prospective study. J Cataract Refract Surg 1998;24:1390-5

18 Riley AF, Malik TY, Grupcheva CN, et al. The Auckland cataract study: comorbidity, surgical techniques, and clinical outcomes in a public hospital service. Br J Ophthalmol 2002;86:185-90.

19 Powe NR, Schein OD, Gieser SC, et al. Synthesis of literature on visual acuity and complications following cataract extraction with intraocular lens implantation. Arch Ophthalmol 1994;112:239-52

20 Natchiar GN, Robin Al, Nalgirkar AR, et al. Posterior capsule tears during extracapsular cataract surgery in India. Arch Ophthalmol 1993;111:706-8.

21 Natchiar GN, Thulasiraj RD, Negrel D, et al. The Madurai intraocular lens study I: A randomized clinical trial comparing complications and vision outcomes of intracapsular cataract extraction and extracapsular cataract extraction with posterior chamber intraocular lens. Am J Ophthalmol 1998; 125:1-13. 